\title{
Tuberculose buccale : à propos d'un cas
}

\author{
Tuberculosis of the oral cavity: a case report
}

AHMED ELFAHSI, ALI ELAYOUBI, MALIK BOULAADAS, NAOUAL NAZIH, LEILA ESSAKALI, MOHAMED KZADRI

\section{RÉSUMÉ}

La tuberculose de la cavité buccale reste rare, son tableau clinique non spécifique. L'étude bactériologique et histopathologique tient un rôle important, permettant de préciser la nature tuberculeuse des lésions. L'évolution est favorable sous traitement médical, maintenant bien codifié.

Un homme de 45 ans, traité pour tuberculose pulmonaire 6 ans auparavant, a présenté un processus ulcérobourgeonnant dans la région rétromolaire gauche. Un orthopantomogramme a objectivé une image lacunaire sur la crête alvéolaire de l'extrémité distale de la branche horizontale gauche de la mandibule ; une biopsie a été réalisée et l'examen histopathologique orienté vers une tuberculose.

L'intérêt de cette présentation est d'attirer l'attention sur la tuberculose que l'on peut retrouver de façon exceptionnelle dans certaines localisations, comme la cavité buccale. Med Buccale Chir Buccale 2007; 13: 83-86.

mots clés: tuberculose, cavité buccale

\section{SUMMARY}

The tuberculosis of oral cavity is rare and its clinical presentation non specific. The bacteriological and histopathological results have an important role, permitting to confirm the tubercular nature of the lesions. The evolution is generally favourable under a medical treatment.

A 45-years old patient was treated for pulmonary tuberculosis, 6 years ago; he is admitted in our formation for an ulcerous tumour in the left retromolar region. A dental planning objectified an osteolysis located on the alveolar ridge of the left posterior mandible. The biopsy concludes to the diagnosis of tuberculosis.

The interest of this presentation is to attract the attention on the tuberculosis, which can be located in some unsuspected regions, and have unusual clinical presentations. Med Buccale Chir Buccale 2007; 13: 83-86.

key words: tuberculosis, oral cavity 
La tuberculose primaire de la cavité buccale est une affection très peu fréquente. Dans la plupart des cas, elle est secondaire à une tuberculose pulmonaire. Dans les pays du tiers monde, où la tuberculose sévit à l'état endémique, elle doit toujours être évoquée dans le diagnostic différentiel de nombreuses lésions. Le balayage par la salive, la grande variété de la flore buccale et la barrière que constitue la muqueuse buccale représentent, pour la cavité buccale, des facteurs de la résistance à Mycobacterium tuberculosis. L'intérêt de cette présentation est d'attirer l'attention sur la tuberculose que l'on peut retrouver dans des localisations devenues exceptionnelles. II faut y penser systématiquement dans certaines situations, principalement dans les régions où la tuberculose est endémique et chez le sujet immunodéprimé.

médecine buccale chirurgie buccale

VOL. $13, \mathrm{~N}^{\circ} 2$ 2007 page 84

\section{OBSERVATION CLINIQUE}

II s'agit d'un homme de 45 ans, tabagique chronique, traité pour une tuberculose pulmonaire 6 ans auparavant. Le début de l'affection remonte à 8 mois : elle s'est manifestée par l'apparition d'une petite formation tumorale siégeant dans la région rétromolaire gauche, augmentant pro-

Figure 1 : Lésion ulcéro-bourgeonnante de la région rétromolaire gauche touchant aussi la muqueuse jugale et le palais mou

The process in the left retromolar region extended to the buccal mucosa and the soft palate

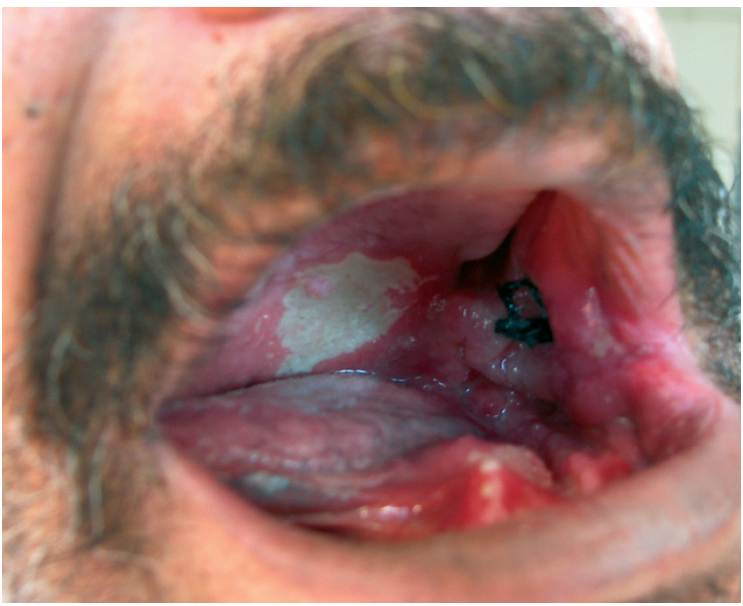

gressivement de taille. Cette lésion était accompagnée de douleurs à la mastication et d'une altération de l'état général avec amaigrissement. L'examen endo-buccal montre un patient édenté avec un processus ulcéro-bourgeonnant, localisé dans la région rétromolaire gauche mais intéressant également la muqueuse jugale et le palais mou (Fig. 1). Les aires ganglionnaires cervicales sont libres, le reste de l'examen sans particularités.

Le bilan biologique est normal excepté une vitesse de sédimentation élevée. La radiographie pulmonaire a trouvé une lésion séquellaire sur les deux apex. L'orthopantomogramme a objectivé une image lacunaire dans la région rétromolaire gauche (Fig. 2). L'intradermo-réaction est positive et mesure $10 \mathrm{~mm}$ de diamètre.

Devant ce tableau clinique et radiologique, le diagnostic de carcinome épidermoïde a été évoqué en premier lieu. Cinq biopsies ont été réalisées : les trois premières montraient des lésions compatibles avec une réaction inflammatoire granulomateuse, alors que les deux dernières ont permis de poser le diagnostic de gingivite granulomateuse d'origine tuberculeuse.

Apres avoir écarté une éventuelle deuxième localisation tuberculeuse; le patient a été mis sous traitement anti-bacillaire (association d'isoniazide,

Figure 2 : Image lacunaire sur la crête alvéolaire de l'extrémité distale de la branche horizontale de la mandibule

Lacunar image on the alveolar ridge of the left posterior mandible

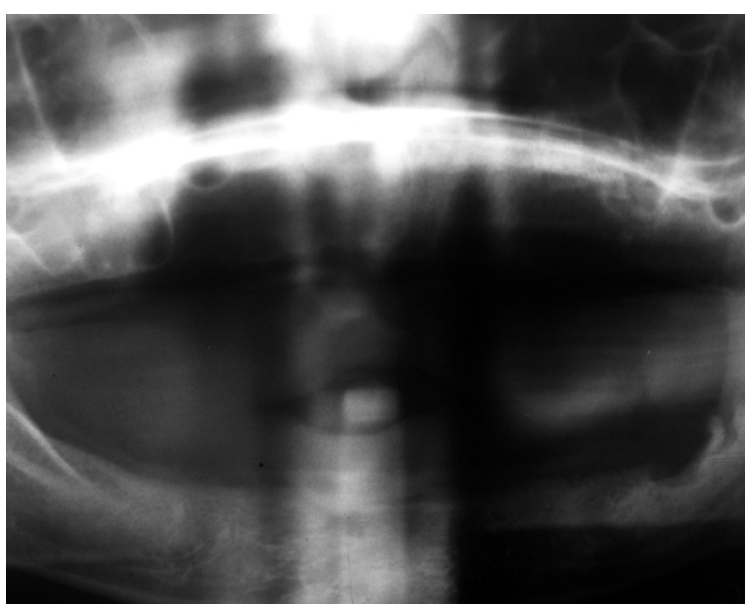


de rifampicine et de pyrazinamide). Une nette amélioration a été constatée après le deuxième mois de traitement; à la fin du traitement, on a constaté la disparition des lésions buccales et une reprise pondérale. Un bilan radiologique de contrôle a été demandé mais il a été impossible de recontacter le patient.

\section{COMMENTAIRES}

La tuberculose est une affection qui sévit encore à l'état endémique au Maroc. Le risque annuel d'infection est de l'ordre de 2 à $5 \%$, soit environ 20 à 50 fois plus important que dans les pays industrialisées. L'incidence annuelle est de l'ordre de 120 nouveaux cas par 100000 habitants. Dans la région cervico-faciale, la tuberculose touche le plus souvent les ganglions lymphatiques. Sa localisation sur la muqueuse buccale est très rare et s'observe surtout chez l'enfant et l'adolescent [1,2]. L'atteinte des os de la face est exceptionnelle: $0,5 \%$ des atteintes osseuses et 3,5\% des ostéites maxillaires ${ }^{[3]}$. Les manifestations cliniques ne sont pas spécifiques et l'aspect radiologique parfois trompeur peuvent orienter vers d'autres pathologies.

La tuberculose endo-buccale se manifeste généralement sous deux formes. Dans le complexe primaire, l'ulcération tuberculeuse ${ }^{[4,5]}$ qui représente le chancre d'inoculation, siège le plus souvent sur les lèvres, et l'adénopathie satellite, unique ou multiple, de taille variable, peut se fis-

\section{RÉFÉRENCES}

1 - Dimitrakopoulos I, Zouloumis L, Lazaridis N, Karakaris D, Trigodinis G, Sichletidis L. Primary tuberculosis of the oral cavity. Oral Surg Oral Med Oral Pathol 1991; 72 : 712-5.

2 - Ver Ma A, Mann SB, Radotra B. Primary tuberculosis of the tongue. Ear Nose Throat J 1989 ; 68 : 718-20.

3 - Gouzien G, Morino JL, Perret JL, Lunven Y. Tuberculose mandibulaire et malaire à propos d'un cas. Rev Stomatol Chir Maxillofac 1991 ; 92 : 112-5. tuliser à la peau. Dans l'autre forme, l'ulcération tuberculeuse touche surtout sur les bords latéraux de la langue. Dans l'ensemble, la gencive représente la deuxième localisation par ordre de fréquence dans la forme ulcéro-végétante; on retrouve souvent la notion d'extraction ou d'un mauvais état bucco-dentaire [6]. La localisation mandibulaire est très peu fréquente [7].

La contamination se fait directement par inhalation de micro-gouttelettes de salive infectée ou par voie ascendante à partir d'un foyer tuberculeux pulmonaire. Le diagnostic, suspecté cliniquement, est confirmé histologiquement par la mise en évidence d'un granulome épithélioïde giganto-cellulaire avec nécrose caséeuse ${ }^{[8,9]}$. La culture est positive dans 50 à $75 \%$ [10]. L'étude histologique permet d'éliminer d'autres pathologies : sarcoïdose, syphilis, carcinome épidermoïde, ulcération traumatique... [11]. Le traitement de la tuberculose est médical, quelle que soit sa localisation, basé essentiellement sur l'association d'antibacillaires selon des protocoles bien définies. Il comporte deux phases. Lors des premiers deux mois, le traitement associe en prise quotidienne : isoniazide (300 $\mathrm{mg})$, rifampicine (600 mg) et pyrazinamide (2 g). Pendant la phase de consolidation qui dure quatre mois au minimum, le traitement comporte soit des prises quotidiennes (isoniazide $300 \mathrm{mg} / \mathrm{j}$ et rifampicine $600 \mathrm{mg} \times 2 / \mathrm{j}$ ) ou bihebdomadaire (isoniazide $900 \mathrm{mg} \times 2 / \mathrm{sem}$. et rifampicine $600 \mathrm{mg} \times 2 / \mathrm{sem}$.) [12].

4 - Favia G, Chiaravalle G, Lacaita MG, Laforgia A, Fina A. La tuberculosi oro-faciale. Analisi generale ed ananatomo-clinica di 35 casi. Minerva Stomatol 1990 ; 73 : 261-8.

5 - Gay C, Buenechea I. Tuberculosis oral: de tres presentaciones casos. Rev Actual Odentoestomatol Esp 1991; 51: 41-4.

6 - Hashimoto Y, Tanioka H. Primary tuberculosis of the tongue: report of a case. J Oral Maxillofac Surg 1989; 47 : 744-6. médecine

buccale chirurgie buccale

VOL. $13, \mathrm{~N}^{\circ} 2$ 2007

page 85 
7 - Fukuda J, Shingo Y, Miyako H. Primary tuberculosis osteomyelitis of the mandibule. Oral Surg Oral Med Oral Pathol 1992 ; 73 : 278-80.

8 - Singhal SK, Dass A, Mohan H, Venkataramana Y. Primary nasal tuberculosis. J Otolaryngol 2002 ; 31 : 60-2.

9 - Hup AK, Haitjema T, Dekuijper G. Primary nasal tuberculosis. Rhinology $2001 ; 39$ : 47-8.

10 - Konishi K, Yaman H, Iguchi H, Nakagawa T, Shibata S, Takayama M, Sunami K, Nakai Y. Study of tuberculosis in the field of otorhinolaryngology in the past 10 years. Acta Otolaryngol 1998 ; 538 (suppl) : 244-9.
11 - Daghfous MH, Nagi S, Ben Hajel H, Robbana A, Khaled A, Hached M, Ben Kheder N. Approche sialoéchographique de la tuberculose primitive des glandes salivaires. A propos de 3 observations. J Radiol 1994 ; $75: 229-32$.

12 - Coulon JP, Piette E. Tuberculose. Encycl Med Chir Stomatol 2001 ; 22047 A 10. médecine buccale chirurgie buccale

VOL. $13, N^{\circ} 2$ 2007

page 86 